\title{
The Action of Instantons with Nut Charge
}

\author{
C.J.Hunter* \\ Department of Applied Mathematics and Theoretical Physics, University of Cambridge, \\ Silver Street, Cambridge CB3 9EW, United Kingdom
}

(6 July 1997)

\begin{abstract}
We examine the effect of a non-trivial nut charge on the action of non-compact four-dimensional instantons with a $U(1)$ isometry. If the instanton action is calculated by dimensionally reducing along the isometry, then the nut charge is found to make an explicit non-zero contribution. For metrics satisfying AF, ALF or ALE boundary conditions, the action can be expressed entirely in terms of quantities (including the nut charge) defined on the fixed point set of the isometry. A source (or sink) of nut charge also implies the presence of a Misner string coordinate singularity, which will have an important effect on the Hamiltonian of the instanton.
\end{abstract}

04.70.Dy, 04.20.-q

Typeset using REVTEX

*email: C.J.Hunter@damtp.cam.ac.uk 


\section{INTRODUCTION}

Since the first publication [1] that proposed a microscopic interpretation of black hole entropy in terms of D-branes, there has been a resurgence of interest in black hole entropy. From the Euclidean quantum gravity point of view [2], this entropy is (in $d$-dimensions) due to the $d-2$ dimensional fixed point set of the imaginary time isometry. However, there are many other types of fixed point sets which could also give rise to entropy.

In this paper we investigate four-dimensional instantons with nut charge. The presence of a nonzero total nut charge means that not only are the fixed point sets more complicated than the simple black hole ones, but also that the solutions will not be asymptotically flat (AF), but rather will be asymptotically locally flat (ALF), or asymptotically locally Euclidean (ALE). The motivation for studying the properties of these instantons is that it is an initial step towards understanding the effect of nut charge on entropy (which will be given in a separate paper [3]).

The outline of the paper is as follows: we begin in section 11 by introducing the gravitational instantons which will serve as examples for the calculations in the rest of the paper. In section [II the action formula for an instanton with a $U(1)$ isometry is dimensionally reduced to an integral over the orbit space of the isometry. Then in section $[\nabla]$ the nut charge, nut potential, and the corresponding Misner string coordinate singularity are described. Some concluding remarks are made in section $\square$, and a few mathematical results used in other sections are given in the appendices.

\section{II. $U(1)$ GRAVITATIONAL INSTANTONS}

Much work has been devoted to the subject of black hole thermodynamics. In general relativity, the non-trivial value for the entropy of a black hole is due to the presence of the fixed point set of the periodic imaginary time Killing vector (the fixed point set is actually the black hole horizon). Although by far the most physically relevant, black hole horizons are just the simplest kind of fixed point set of a $U(1)$ isometry. More generally, in four dimensions such fixed point sets can be classified into zero-dimensional nuts and twodimensional bolts, which may or may not have a nut charge (the black hole horizons that have been considered so far have zero nut charge). In this section we introduce examples of gravitational instantons with nuts, or with bolts with nut charge, and calculate their actions.

When written in terms of a single coordinate system, metrics with nut charge are found to have Misner strings, two-dimensional coordinate singularities, which are the result of trying to use stationary coordinates for metrics that are not a product near infinity. The presence of a Misner string means that the metrics are not asymptotically flat, and hence we cannot use flat space as a background for the action and Hamiltonian calculations (a background metric is necessary in order to obtain a finite result), but instead must consider a background with the same asymptotic behaviour. In our case, this will turn out to be the multi-Taub-NUT [4] or the ALE [5] metrics.

The spaces that we will consider are the oriented manifolds $\mathcal{M}$, with Riemannian metrics $g_{\mu \nu}$, that have a $U(1)$ isometry with group action $\mu_{\tau}: \mathcal{M} \rightarrow \mathcal{M}$, where the group parameter is taken to be the coordinate $\tau$ (and $\xi=\partial_{\tau}$ is the corresponding Killing vector) which has period $\beta$. In many cases (but not all), $\tau$ will be an imaginary time coordinate obtained by 
analytically continuing a Lorentzian metric to a Euclidean one. The classification of such metrics in terms of their fixed point sets was first considered by Gibbons and Hawking [6] in 1979.

The $U(1)$ isometry has a fixed point at $p$ if $\xi=0$ at $p$. This gives rise to an isometry $\mu_{\tau} *$ of the tangent space at $p$, which is generated by the covariant derivative of the Killing vector, i.e., the antisymmetric matrix $\xi_{\mu ; \nu}$. We are interested in the rank of $\xi_{\mu ; \nu}$, because it tells us the number of directions that are invariant under $\mu_{\tau} *$. An antisymmetric $4 \times 4$ matrix has rank (besides the trivial rank of zero) two or four.

If the rank is two, then there is a local two-dimensional surface around $p$ in which $\mu_{\tau}$ will be the identity (and hence the points on this surface will be fixed). The surface is generated by the vectors which are fixed by $\mu_{\tau} *$. If we move in the other directions, then $\mu_{\tau}$ is not the identity, since $\mu_{\tau} *$ does not fix vectors that point in those directions. Thus, we have a two-dimensional fixed point set, called a bolt. The canonical example is the Euclidean Schwarzschild metric, where the horizon two-surface is a bolt of the imaginary time isometry.

If the rank is four, then we see that there are no directions in which $\mu_{\tau}$ is the identity. Thus we have an isolated, or zero-dimensional fixed point set, called a nut, after its canonical example, the Euclidean Taub-NUT instanton, which is described further in section 【IC.

We would like to consider the manifold $\mathcal{M}$ in terms of a fibre bundle structure based on the $U(1)$ isometry, that is, where the fibres are the orbits of $\xi$. In order to do so, we cannot include the fixed points of $\xi$, since they have degenerate orbits. Thus, we want to consider $\mathcal{M}^{\prime}=\mathcal{M}-\{$ fixed points $\}$, that is the manifold minus its fixed point set. Once we have deleted the degenerate fibres, we can consider $\mathcal{M}^{\prime}$ as a $U(1)$ principal bundle over the orbit space $\Xi$. The fact that we want to work with $\mathcal{M}^{\prime}$ rather than $\mathcal{M}$ will be very important in our calculations.

Given an instanton, we are interested in calculating its action, since this is an important quantity for describing the thermodynamics of the system, and also gives a measure of the probability for a decay mediated by the instanton to occur. In order to obtain a finite value for the action of a non-compact spacetime, it is necessary to define a background spacetime, which may be considered as a ground state or reference spacetime, with respect to which the spacetime under consideration may be compared [0, [8]. The Euclidean action is then given by円

$$
I=-\frac{1}{16 \pi} \int_{\mathcal{M}} d^{4} x \sqrt{g} R-\frac{1}{8 \pi} \int_{\partial \mathcal{M}} d^{3} x \sqrt{b}[\Theta(b)-\Theta(\tilde{b})]
$$

where $\mathcal{M}$ is a compact region of the spacetime, with boundary $\partial \mathcal{M}$ (which we let tend to infinity), $b_{\mu \nu}$ is the induced metric on $\partial \mathcal{M}$, and $\Theta$ is the trace of the extrinsic curvature of $\partial \mathcal{M}$ in $\mathcal{M}$. A tilde will be used throughout to indicate a term which is defined on the background spacetime, however, for simplicity we will generally omit the background term from calculations. In the limit that $\partial \mathcal{M}$ goes to infinity, a finite and well-defined action will be obtained. Note that for solutions of the vacuum Einstein fields equations, such as the instantons discussed later in this section, the action will come entirely from the surface

\footnotetext{
${ }^{1}$ Additional terms due to the non-orthogonality of the boundaries, described in 8$]$, have been omitted for clarity.
} 
integral (the background is assumed to be a solution of the field equations, and hence never has a volume contribution). For a background to be suitable for a given spacetime, it must be possible to match the metric that it induces on the hypersurface $\partial \mathcal{M}$, to the metric induced by the spacetime on $\partial \mathcal{M}$ (at least to a sufficiently high asymptotic order, so that a difference in the induced metrics does not affect the surface integrals). A compact spacetime does not require the use of a background.

In order to calculate the action, we would like to simplify the integration by factoring out the isometry. We can accomplish this by two possible routes - we can either dimensionally reduce along the orbits of the isometry, or we can perform a Hamiltonian decomposition. In this paper we are only interested in the dimensional reduction method, however the Hamiltonian decomposition is crucial in calculating the entropy of the instanton [3].

In order to perform dimensional reduction, we write the metric in Kaluza-Klein form,

$$
d s^{2}=V\left(d \tau+2 A_{i} d x^{i}\right)^{2}+\frac{1}{V} \gamma_{i j} d x^{i} d x^{j},
$$

and then the three-dimensional orbit space $\Xi$ is characterized by a metric $\gamma_{i j}$, a vector field $A_{i}$, and a scalar field $V$. The fixed points of $\xi$ will occur at the zeros of $V$. As will be shown in section III, one of the principle advantages of the Kaluza-Klein method is that we can obtain expressions for the action of a spacetime in terms of fixed point quantities, such as its area, nut charge and nut potential.

In order to dimensionally reduce along the isometry we need a fibre bundle structure, and hence we need to consider $\mathcal{M}^{\prime}$ rather than $\mathcal{M}$. This will lead to a boundary in $\Xi$ around each fixed point set, and additional contributions to the action from the regions of the manifold that we have discarded. The action of a nut is zero, but if $\mathcal{P}$ is a two-surface to be excised from the manifold $\mathcal{M}$, then it will have a nonzero action. To calculate this action we need to consider a family of tubular neighbourhoods, $\mathcal{T}_{r}$, of radius $r$ around $\mathcal{P}$. The action of $\mathcal{P}$ is then obtained by taking the limit as $r \rightarrow 0$ of the actions of the tubular neighbourhoods. The action of $\mathcal{T}_{r}$ is entirely due to the integral of the trace of the second fundamental form over the hypersurface $\partial \mathcal{T}_{r}$, which is related to the radial derivative of the boundary volume,

$$
I=-\frac{1}{8 \pi} \lim _{r \rightarrow 0} \int_{\partial \mathcal{T}_{r}} d^{3} x \sqrt{b} \Theta(b)=-\frac{1}{8 \pi} \lim _{r \rightarrow 0} \frac{\partial}{\partial r} \operatorname{vol}\left(\partial \mathcal{T}_{r}\right),
$$

where $b_{\mu \nu}$ is the induced metric on $\partial \mathcal{T}_{r}$, and $\Theta$ is its extrinsic curvature. Using a Taylor series expansion about $\mathcal{P}$ of the hypersurface volume form, we can calculate the boundary volume to be

$$
\operatorname{vol}\left(\partial \mathcal{T}_{r}\right)=2 \pi r \operatorname{vol}(\mathcal{P})+r^{3} \int_{\mathcal{P}} d^{2} x F(x)+\mathcal{O}\left(r^{5}\right)
$$

where $F(x)$ depends only on the Riemann tensor, and hence is independent of $r$. Differentiating with respect to the radial distance yields

$$
\frac{\partial}{\partial r} \operatorname{vol}\left(\partial \mathcal{T}_{r}\right)=2 \pi \operatorname{vol}(\mathcal{P})+2 r^{2} \int_{\mathcal{P}} d^{2} x F(x)+\mathcal{O}\left(r^{4}\right)
$$

and hence the action is

$$
I=-\frac{1}{8 \pi} \lim _{r \rightarrow 0} \frac{\partial}{\partial r} \operatorname{vol}\left(\partial \mathcal{T}_{r}\right)=-\frac{1}{4} \operatorname{vol}(\mathcal{P})
$$


Thus a bolt makes a contribution to the action of

$$
I=-\frac{\mathcal{A}}{4}
$$

where $\mathcal{A}$ is the area of the two-surface. This contribution must be included when $\mathcal{M}^{\prime}$, rather than $\mathcal{M}$ is used to calculate the action. Note that if the two-surface is non-compact, that is, it extends out to infinity, then it must have a counterpart in the background spacetime, since the behaviour at infinity which must be matched between the two metrics. In this case, there will be a contribution to the action of

$$
I=-\frac{\Delta \mathcal{A}}{4}
$$

where $\Delta \mathcal{A}$ is the difference in area between the two-surface in the spacetime and in the background.

\section{A. Asymptotic Classes}

The asymptotic behaviour of instantons that we are interested can be divided into three classes:

Asymptotically Flat (AF) The boundary at infinity is an $S^{1}$ bundle over an $S^{2}$, where the $S^{1}$ fibers approach a constant length. Such bundles are labeled by their first Chern number, $c_{1}$. If $c_{1}$ is zero, the metric is said to be $\mathrm{AF}$, has boundary topology $S^{1} \times S^{2}$, and approaches the metric

$$
d s^{2} \sim\left(1-\frac{2 M}{r}\right) d \tau^{2}+\left(1+\frac{2 M}{r}\right) d \mathcal{E}_{3}^{2}+\mathcal{O}\left(r^{-2}\right)
$$

at large radius. $d \mathcal{E}_{3}^{2}$ is the flat metric on three-dimensional Euclidean space, and the periodicity of $\tau$ is not fixed. The Kerr instanton is AF.

Asymptotically Locally Flat (ALF) If $c_{1}=n$, then the metric is ALF, the boundary topology is $S^{3} / \mathcal{Z}_{n}$, and it asymptotically approaches the metric

$$
d s^{2}=\left(1-\frac{2 M}{r}\right)(d \tau+2 a N \cos \theta d \phi)^{2}+\left(1+\frac{2 M}{r}\right) d \mathcal{E}_{3}^{2}+\mathcal{O}\left(r^{-2}\right),
$$

where the periodicity of $\tau$ is $8 \pi a N / n$. Both Taub-NUT and Taub-Bolt are ALF metrics.

Asymptotically Locally Euclidean (ALE) A metric is ALE if the manifold has a boundary $S^{3} / \Gamma$ at infinity, where $\Gamma$ is a discrete subgroup of $S O(4)$ with a free action on $S^{3}$, and the metric asymptotically approaches

\footnotetext{
${ }^{2}$ In [9] an alternate asymptotic form of the ALE metric is given, however, since we are specifically interested in vacuum solutions, we can use the more restrictive metric given here.
} 


$$
d s^{2}=\left(1+\frac{M}{r^{4}}\right) d \mathcal{E}_{4}^{2}+\mathcal{O}\left(r^{-5}\right)
$$

where $d \mathcal{E}_{4}^{2}$ is the four-dimensional Euclidean metric. The Eguchi-Hanson instanton is an example of an ALE metric.

Asymptotically Euclidean (AE) metrics are simply ALE with $\Gamma=1$. Thus, they are really just a special case of an ALE space, and we will not be treated as a separate class. Note that from the proof of the positive action conjecture [10], we know that the only AE instanton is four-dimensional Euclidean space. We will use the term ALL spaces to refer to the combined class of AF, ALF and ALE metrics.

As mentioned ealier, in order to obtain a finite result for the action, we need to use a background metric. Thus, it would be useful to derive the asymptotic form of the background metrics for the asymptotic classes presented above. For AF metrics, flat space is the background. We need to scale both the imaginary time and the radial coordinate in order to match flat space to the metric (2.8) on a hypersurface of constant radius $R$. The matched background metric is then

$$
d \tilde{s}^{2}=\left(1-\frac{2 M}{R}\right) d \tau^{2}+\left(1+\frac{2 M}{R}\right) d \mathcal{E}_{3}^{2} .
$$

For ALF spaces, the background is the multi-Taub-NUT metric, which has the same asymptotic form as (2.9), but with $\tilde{M}=\tilde{N}$. We want to match this background metric to the asymptotic metric (2.9) on a hypersurface of constant radius $R$. We can do this by setting the parameter $\tilde{N}=n(R) N$, and scaling both the imaginary time $\tilde{\tau}=n(R) \tau$, and the radial coordinate $\tilde{r}=\lambda(R) r$,

$$
d \tilde{s}^{2} \sim n(R)^{2}\left(1-\frac{2 a N n(R)}{\lambda(R) r}\right)(d \tau+2 a N \cos \theta)^{2}+\lambda(R)^{2}\left(1+\frac{2 N n(R)}{r \lambda(R)}\right) d \mathcal{E}_{3}^{2} .
$$

If we expand $n(R)$ and $\lambda(R)$ in a Taylor series in $R^{-1}$, then we can match the metrics to the correct order by setting

$$
n(R)=1-\frac{M-N}{R} \text { and } \quad \lambda(R)=1+\frac{M-N}{R} .
$$

We can then simplify the metric (2.12) by keeping only terms which are first order in $R^{-1}$ and $r^{-1}$,

$$
d \tilde{s}^{2} \sim\left(1-\frac{2 N}{r}-\frac{2(M-N)}{R}\right)(d \tau+2 a N \cos \theta)^{2}+\left(1+\frac{2 N}{r}+\frac{2(M-N)}{R}\right) d \mathcal{E}_{3}^{2} .
$$

Finally, we want to consider the background space for an ALE instanton. This will simply be four-dimensional Euclidean space (no scaling is necessary),

$$
d \tilde{s}^{2}=d \mathcal{E}_{4}^{2}
$$

In the remainder of this section we introduce the Kerr, Taub-NUT, Taub-Bolt, and EguchiHanson instantons which we will use as examples throughout the paper. 


\section{B. Kerr}

A Euclidean Kerr metric can be obtained by Wick rotating both the time, and the rotation parameter of the Lorentzian Kerr solution,

$$
\begin{aligned}
d s^{2}= & V\left[d \tau-2 \frac{a M r \sin ^{2} \theta}{\Delta+a^{2} \sin ^{2} \theta} d \phi\right]^{2} \\
& +\frac{1}{V}\left[\frac{\Delta+a^{2} \sin ^{2} \theta}{\Delta} d r^{2}+\left(\Delta+a^{2} \sin ^{2} \theta\right) d \theta^{2}+\Delta \sin ^{2} \theta d \phi^{2}\right]
\end{aligned}
$$

where

$$
V=1-\frac{2 M r}{r^{2}-a^{2} \cos ^{2} \theta} \quad \text { and } \quad \Delta=r^{2}-2 M r-a^{2}
$$

The metric is asymptotically flat, since the rotation parameter, $a$, only enters to second order in the metric. The instanton is regular in the region $r \geq r_{+}$, where

$$
r_{+}=M+\sqrt{M^{2}+a^{2}}
$$

is the horizon of the black hole, and the bolt of the co-rotating Killing vector $\partial_{\tau}+\Omega \partial_{\phi}$, where

$$
\Omega=\frac{a}{r_{+}^{2}-a^{2}}=\frac{a}{2 M r_{+}}
$$

The points $(\tau, r, \theta, \phi)$ must be identified with $(\tau+2 \pi \gamma, r, \theta, \phi+2 \pi \gamma \Omega)$, where

$$
\gamma=\frac{2 M r_{+}}{\sqrt{M^{2}+a^{2}}}
$$

If instead of the co-rotating vector, we simply want to consider $\xi=\partial_{\tau}$, then the fixed point set is a nut and anti-nut (an anti-nut is a nut with negative nut charge) pair located at $r=r_{+}$and $\theta=0, \pi$, that is, at the poles of the horizon.

Because of the relation between $\tau$ and $\phi$, the coordinate ranges are somewhat complicated. The total area of the $\tau-\phi$ plane is $4 \pi \gamma$. We can take $\phi$ to be periodic with period $2 \pi$, but then $\tau$ is not a periodic coordinate (except when accompanied by a translation in $\phi)$, but simply has range $[0,2 \pi \gamma]$. If we assume that $\gamma \Omega=q / p$, where $q$ and $p$ are integers, then $\tau$ can be taken to be a periodic coordinate with period $2 \pi \gamma p$, in which case $\phi$ is no longer periodic (except when accompanied by a translation in $\tau$ ), but has a range $[0,2 \pi / p]$. Since we want $\partial_{\tau}$ to be a $U(1)$ isometry, we restrict our attention to the latter case.

The action calculation is easy. Since Kerr is asymptotically flat, the background is a scaled flat metric

$$
d s^{2}=\left(1-\frac{2 M}{R}\right) d \tau^{2}+d r^{2}+r^{2} d \theta^{2}+r^{2} \sin ^{2} \theta d \phi^{2},
$$

which matches (to sufficient order) the Kerr metric on a boundary of radius $R$. The extrinsic curvature of the Kerr metric is 


$$
\Theta(b)=\frac{2}{R}-\frac{M}{R^{2}}+\mathcal{O}\left(R^{-3}\right)
$$

while the background value is

$$
\Theta(\tilde{b})=\frac{2}{R}
$$

Thus, the action is simply

$$
I=-\frac{1}{8 \pi} \int d \tau d \phi \int_{0}^{\pi} d \theta R^{2} \sin \theta\left(-\frac{M}{R^{2}}\right)=\pi \gamma M
$$

\section{Taub-NUT and Taub-Bolt}

The self-dual Taub-NUT instanton [ [4] is a non-compact solution of the field equations given by the metric

$$
d s^{2}=V(r)(d \tau+2 N \cos \theta d \phi)^{2}+\frac{1}{V(r)} d r^{2}+\left(r^{2}-N^{2}\right)\left(d \theta^{2}+\sin ^{2} \theta d \phi^{2}\right)
$$

where $V(r)$ is

$$
V_{T N}(r)=\frac{r-N}{r+N}
$$

In order to make the solution regular, we consider the region $r \geq N$, and let the period of $\tau$ be $8 \pi N$. It is asymptotically locally flat, since a boundary at large radius $r$ is a squashed $S^{3}$ - it is an $S^{1}$ bundle with constant circumference $\beta$, (parameterized by $\tau$ ), over an $S^{2}$ which expands with the radius $r$, (parameterized by $(\theta, \phi)$ ). There is a zero of $V$ at $R=N$, and hence a fixed point set. However, the $S^{2}$ parameterized by $(\theta, \phi)$ vanishes at the origin, so the fixed point set is zero-dimensional, that is, a nut.

The Taub-Bolt instanton [11] is a non-self-dual, non-compact solution of the vacuum Euclidean Einstein equations, and is also given by the metric (2.25). However, the function $V(r)$ is different,

$$
V_{T B}(r)=\frac{(r-2 N)(r-N / 2)}{r^{2}-N^{2}} .
$$

The solution is regular if we consider the region $r \geq 2 N$, and let $\tau$ have period $\beta=8 \pi N$. Asymptotically, the Taub-Bolt instanton has the same behaviour, i.e., ALF, as the Taub-

NUT instanton. $V$ has a zero, and hence $\xi$ has a fixed point set, at $r=2 N$. Since the $S^{2}$ does not vanish there, the fixed point set is two-dimensional, and hence a bolt. It has area $12 \pi N^{2}$. Note that it is not possible to define fermion fields on the Taub-Bolt instanton because it has no spin structure, however, it is possible to give it a generalized spin structure by coupling the fermions to an external field [12.

Both the Taub-NUT and Taub-Bolt instantons are obtained by Wick rotating the Lorentzian Taub-NUT solution. While the Lorentzian solution has two independent parameters, $M$ and $N$, the instantons only have one, since $M$ is fixed in terms of $N$ in order 
to avoid a conical singularity at the origin. The difference in $V(r)$ between the Taub-NUT and Taub-Bolt solutions arises from the two different ways of fixing $M$ in terms of $N$.

We now want to calculate the action of the Taub-Bolt instanton. The most natural background to consider is the Taub-NUT solution". In order to calculate the action, we want to consider the compact region $\mathcal{M}$ inside a boundary of radius $R$. The Taub-NUT metric, as given by equation (2.25), does not match the Taub-Bolt metric correctly on the boundary. However, if we scale $\tilde{r}, \tilde{\tau}$ and $\tilde{N}$ in terms of $R$ and $N$, then we can match the the two metrics on the boundary. The required transformation leaves the radial coordinate unchanged (i.e., we use the boundary of radius $R$ in the background spacetime), while the nut charge is given by $\tilde{N}=N m$, where

$$
m=\left(1-\frac{N}{4 R}\right)
$$

The variable $\tilde{\tau}$ is transformed by

$$
\tilde{\tau} \rightarrow m \tilde{\tau}
$$

so that the new $\tilde{\tau}$ coordinate will have period $8 \pi N$, identical to the period of $\tau$ in the Taub-Bolt metric. Thus, the correctly matched Taub-NUT metric is

$$
d s^{2}=m^{2} \tilde{V}_{T N}(d \tilde{\tau}+2 N \cos \tilde{\theta} d \tilde{\phi})^{2}+\frac{1}{\tilde{V}_{T N}} d r^{2}+\left(\tilde{r}^{2}-m^{2} N^{2}\right)\left(d \tilde{\theta}^{2}+\sin ^{2} \tilde{\theta} d \tilde{\phi}^{2}\right)
$$

where

$$
\tilde{V}_{T N}=\frac{r-m N}{r+m N}
$$

If we calculate the trace of the extrinsic curvature of the three-sphere of radius $R$ in the Taub-Bolt instanton, we obtain (to sufficient order)

$$
\Theta(b)=\frac{2}{R}-\frac{5}{4} \frac{N}{R^{2}}+\mathcal{O}\left(\frac{1}{R^{3}}\right) .
$$

A similar calculation in the background Taub-NUT solution yields

$$
\Theta(\tilde{b})=\frac{2}{R}-\frac{N}{R^{2}}+\mathcal{O}\left(\frac{1}{R^{3}}\right)
$$

Thus, the infinite terms in the action (of order $R^{-1}$ ) will cancel when the two quantities are subtracted. Calculating the action, we obtain

$$
I=-\frac{1}{8 \pi} \int_{\partial \mathcal{M}} d^{3} x \sqrt{b}[\Theta(b)-\Theta(\tilde{b})]=\pi N^{2} .
$$

\footnotetext{
${ }^{3}$ In reference [13] it is suggested that the background should be $\mathcal{R}^{3} \times S^{1}$. However, this background is appropriate only for metrics that are asymptotically flat, and not for the more general class of ALF metrics, because one cannot match the metrics on the boundary at infinity.
} 
In [14], the actions of the Taub-Bolt and Taub-Nut instantons are given as $5 \pi N^{2}$, and $4 \pi N^{2}$ respectively. These calculations are incorrect because they merely discard the "flat space' term, $2 / R$, from equations (2.32) and (2.33) in order to obtain a finite value for the action. Simply discarding the divergent term is equivalent to a performing background subtraction using a reference spacetime that either does not match the instantons on the boundary, or does not satisfy the field equations, and hence is not as physically relevant as our method of calculating the action.

\section{Eguchi-Hanson}

A non-compact instanton which is a limiting case of the Taub-NUT solution is the Eguchi-Hanson metric [15],

$$
d s^{2}=\left(1-\frac{N^{4}}{r^{4}}\right)\left(\frac{r}{8 N}\right)^{2}(d \tau+4 N \cos \theta d \phi)^{2}+\left(1-\frac{N^{4}}{r^{4}}\right)^{-1} d r^{2}+\frac{1}{4} r^{2} d \Omega^{2},
$$

which has one free parameter $N$. The instanton is regular if we consider the region $r \geq N$, and we let $\tau$ have period $8 \pi N$. Unlike the Taub-Bolt and Taub-NUT instantons, the EguchiHanson metric is asymptotically locally Euclidean, because as we take $r$ to infinity, the boundary is an $S^{3}$ whose radius is proportional to $r$, as opposed to the squashed $S^{3}$ of the Taub-Bolt and Taub-NUT solutions, where one direction reaches a finite size. In addition, since the range of $\tau / 4 N$ is $2 \pi$ and not $4 \pi$, a surface of constant $r$ is $S^{3} / \mathcal{Z}_{2}$, rather than $S^{3}$, that is, it is a three-sphere with antipodal points identified. $\xi$ has a fixed point at $r=N$, which is a bolt of area $\pi N^{2}$.

If we want to calculate the action of the spacetime, then since it is non-compact we need to specify a background. Somewhat surprisingly, it turns out that if we are given an EguchiHanson metric with parameter $N$, then any other Eguchi-Hanson metric with parameter $M$ will be a suitable background. This implies that the action must be independent of the parameter $N$, and since this is the only parameter in the problem, that the action must be zero (when the background subtraction is made).

In order to match the two metrics on a surface of constant radius $R$, we must rescale the imaginary time coordinate in the background,

$$
\tilde{\tau} \rightarrow \frac{N}{M} \tilde{\tau}
$$

so that it has the same period, $8 \pi N$, as the solution that we are matching to. The metric is then

$$
d s^{2}=\left(1-\frac{M^{4}}{\tilde{r}^{4}}\right)\left(\frac{\tilde{r}}{8 N}\right)^{2}(d \tilde{\tau}+4 N \cos \tilde{\theta} d \tilde{\phi})^{2}+\left(1-\frac{M^{4}}{\tilde{r}^{4}}\right)^{-1} d \tilde{r}^{2}+\frac{1}{4} \tilde{r}^{2} d \tilde{\Omega}^{2} .
$$

Note that while the match between the metrics is not perfect on a boundary of radius $R$, it is sufficiently close so that any difference is unimportant as $R$ is taken to infinity.

If we calculate the trace of the second fundamental forms we obtain

$$
\Theta=\frac{3 R^{4}-N^{4}}{R^{3} \sqrt{R^{4}-N^{4}}}=\frac{3}{R}+\frac{N^{4}}{2 R^{5}}+\mathcal{O}\left(\frac{1}{R^{9}}\right),
$$


for the metric, and

$$
\tilde{\Theta}=\frac{3 R^{4}-M^{4}}{R^{3} \sqrt{R^{4}-M^{4}}}=\frac{3}{R}+\frac{M^{4}}{2 R^{5}}+\mathcal{O}\left(\frac{1}{R^{9}}\right)
$$

for the background. When we integrate to calculate the action, the infinite contributions from the different metrics cancel, and since the area element is proportional to $r^{3}$, the integral vanishes as the boundary is taken to infinity,

$$
I=-\frac{1}{8 \pi} \int_{\partial \mathcal{M}} d^{3} x \sqrt{b}[\Theta(b)-\Theta(\tilde{b})]=0 .
$$

We could also use flat Euclidean space with antipodal points identified as a background metric. In appropriate coordinates, the metric is

$$
d s^{2}=\frac{r^{2}}{(8 N)^{2}}(d \tau+4 N \cos \theta d \phi)^{2}+d r^{2}+\frac{1}{4} r^{2} d \Omega^{2},
$$

where the period of $\tau$ is $8 \pi N$. This corresponds to setting $M=0$ in the previous example. The extrinsic curvature is

$$
\tilde{\Theta}=\frac{3}{R}
$$

and hence the action will still be zero.

\section{DIMENSIONALLY REDUCED ACTION}

We will now dimensionally reduce the action integral along the orbits of the $U(1)$ isometry. The advantage of this decomposition is that it allows us to express the action in terms of geometric properties of the fixed point sets of the isometry. For example, we will show that for ALL metrics the action can be expressed as

$$
\bar{I}=\frac{\beta}{4} \sum_{\mathrm{fps}} \psi \mathcal{N}+\sum_{\text {bolts }} \frac{A}{4},
$$

where the subscript fps implies a sum over the fixed point set, and bolts implies a sum over only the bolts. $\mathcal{N}$ and $\psi$ are the nut charge and nut potential, which will be defined later in this section, while $A$ is the area of the bolt. This expression for the action can then be used to obtain a formula for the Komar mass of the instanton,

$$
M_{\text {Komar }}=\frac{1}{2} \sum_{\mathrm{fps}} \psi \mathcal{N}+\frac{1}{4 \pi} \sum_{\text {bolts }} \kappa A,
$$

where $\kappa$ is the surface gravity of the bolt.

We begin by calculating a general expression for the action of a non-compact instanton with a boundary at infinity. The Euclidean action is

$$
I=-\frac{1}{16 \pi} \int_{\mathcal{M}} d^{4} x \sqrt{g} R(g)-\frac{1}{8 \pi} \int_{\partial \mathcal{M}} d^{3} x \sqrt{b} \Theta(b)
$$


(where we are implicitly assuming a background subtraction). However, in order to dimensionally reduce, we must be integrating over the manifold $\mathcal{M}^{\prime}$, rather than $\mathcal{M}$. Thus, we have to introduce internal boundaries around each connected component of the fixed point set, excise the fixed point set from our domain of integration and then include the action of these excised regions separately (as shown in section [I], for a bolt the action is minus one-quarter of its area, while for a nut it is zero). Hence, we want to write the action as

$$
I=-\frac{1}{16 \pi} \int_{\mathcal{M}^{\prime}} d^{4} x \sqrt{g} R(g)-\frac{1}{8 \pi} \int_{\partial \mathcal{M}^{\prime}} d^{3} x \sqrt{b} \Theta(b)-\sum_{\text {bolts }} \frac{A}{4} .
$$

We now write the metric in Kaluza-Klein form,

$$
d s^{2}=e^{2 \sigma}\left(d \tau+2 A_{i} d x^{i}\right)+e^{-2 \sigma} \gamma_{i j} d x^{i} d x^{j},
$$

and note that the volume integrand can be expressed in terms of orbit space variables as

$$
\sqrt{g} R(g)=\sqrt{\gamma}\left[R(\gamma)+2 D^{2} \sigma-2(D \sigma)^{2}-e^{4 \sigma} F^{2}\right]
$$

where $F_{i j}$ is the field strength associated with the one-form potential $A_{i}$,

$$
F_{i j}=\partial_{i} A_{j}-\partial_{j} A_{i}
$$

Thus, the volume contribution to the action is

$$
I_{\mathrm{vol}}=-\frac{1}{16 \pi} \int_{\mathcal{M}^{\prime}} d^{4} x \sqrt{g} R(g)=-\frac{\beta}{16 \pi} \int_{\Xi} d^{3} x \sqrt{\gamma}\left[R(\gamma)+2 D^{2} \sigma-2(D \sigma)^{2}-e^{4 \sigma} F^{2}\right] .
$$

We can integrate the second derivative term to obtain

$$
I_{\mathrm{vol}}=-\frac{\beta}{16 \pi} \int_{\Xi} d^{3} x \sqrt{\gamma}\left[R(\gamma)-2(D \sigma)^{2}-e^{4 \sigma} F^{2}\right]-\frac{\beta}{8 \pi} \int_{\partial \Xi} d^{2} x \sqrt{c} v^{\mu} D_{\mu} \sigma,
$$

where $v^{\mu}$ is the unit normal to the boundary.

The surface integral contribution to the action is

$$
I_{\text {surf }}=-\frac{1}{8 \pi} \int_{\partial \mathcal{M}^{\prime}} d^{3} x \sqrt{b} \Theta(b) .
$$

We can express the integrand in terms of orbit space variables,

$$
\sqrt{b} \Theta(b)=\sqrt{c} \Theta(c)-\sqrt{c} v^{\mu} \partial_{\mu} \sigma
$$

where $c_{a b}$ is the induced metric on the boundary in the orbit space. Thus the surface term can be written as

$$
I_{\text {surf }}=-\frac{\beta}{8 \pi} \int_{\partial \Xi} d^{2} x \sqrt{c} \Theta(c)+\frac{\beta}{8 \pi} \int_{\partial \Xi} d^{2} x \sqrt{c} v^{\mu} \partial_{\mu} \sigma .
$$

If we recombine the volume and surface terms (and include the action of the excised regions), we find that the action is given by 


$$
I=-\frac{\beta}{16 \pi} \int_{\Xi} d^{3} x \sqrt{\gamma}\left[R(\gamma)-2(D \sigma)^{2}-e^{4 \sigma} F^{2}\right]-\frac{\beta}{8 \pi} \int_{\partial \Xi} d^{2} x \sqrt{c} \Theta(c)-\sum_{\text {bolts }} \frac{A}{4} .
$$

Thus, we see that we have a volume contribution which is no longer trivially zero for solutions of the field equations, a curvature contribution from the boundary, and a contribution from the area of the bolts. Consider the extrinsic curvature term on the boundary of the fixed point set. The curvature integral on a nut is zero, while on a bolt, as shown in appendix $\mathrm{A}$,

$$
-\frac{\beta}{8 \pi} \int_{\text {bolt }} d^{2} x \sqrt{c} \Theta(c)=\frac{A}{2} .
$$

Thus, we can eliminate the extrinsic curvature term on the fixed point set boundary, and write the action as

$$
I=-\frac{\beta}{16 \pi} \int_{\Xi} d^{3} x \sqrt{\gamma}\left[R(\gamma)-2(D \sigma)^{2}-e^{4 \sigma} F^{2}\right]-\frac{\beta}{8 \pi} \int_{\infty} d^{2} x \sqrt{c} \Theta(c)+\sum_{\text {bolts }} \frac{A}{4},
$$

where the integral over $\infty$ means the boundary of $\Xi$ at infinity.

We now want to dualize the two-form field strength, $F_{i j}$, and express the action in terms of the dual field strength,

$$
H_{i}=\frac{1}{2 \sqrt{\gamma}} \gamma_{i k} \epsilon^{k l m} F_{l m}
$$

Since $F^{2}=2 H^{2}$, we can rewrite the action as

$$
I=-\frac{\beta}{16 \pi} \int_{\Xi} d^{3} x \sqrt{\gamma}\left[R(\gamma)-2(D \sigma)^{2}-2 e^{4 \sigma} H^{2}\right]-\frac{\beta}{8 \pi} \int_{\infty} d^{2} x \sqrt{c} \Theta(c)+\sum_{\text {bolts }} \frac{A}{4} .
$$

However, this action does not reproduce the field equations because it does not account for the Bianchi identity, $\partial_{[i} F_{j k]}=0$, which the field strength satisfies (since it is an exterior derivative, $\left.F_{i j}=\partial_{[i} A_{j]}\right)$. The Bianchi identity is equivalent to a vanishing divergence of $H_{i}$,

$$
D_{i} H^{i}=0 .
$$

Since this equation does not arise by varying the action, we must consider a different action which contains a Lagrange multiplier $\psi$ (called the nut potential) which enforces this constraint,

$$
\begin{aligned}
I^{\prime}= & -\frac{\beta}{16 \pi} \int_{\Xi} d^{3} x \sqrt{\gamma}\left[R(\gamma)-2(D \sigma)^{2}-2 e^{4 \sigma} H^{2}-\psi D_{i} H^{i}\right] \\
& -\frac{\beta}{8 \pi} \int_{\infty} d^{2} x \sqrt{c} \Theta(c)+\sum_{\text {bolts }} \frac{A}{4} .
\end{aligned}
$$

If we vary $H^{i}$, then we obtain the nut potential equation of motion

$$
4 e^{4 \sigma} H_{i}=D_{i} \psi
$$

We can use this equation to eliminate $H_{i}$ from the action, 


$$
\begin{aligned}
I^{\prime}= & -\frac{\beta}{16 \pi} \int_{\Xi} d^{3} x \sqrt{\gamma}\left[R(\gamma)-2(D \sigma)^{2}-\frac{1}{8} e^{-4 \sigma}(D \psi)^{2}-\frac{1}{4} \psi D_{i}\left(e^{-4 \sigma} D^{i} \psi\right)\right] \\
& -\frac{\beta}{8 \pi} \int_{\infty} d^{2} x \sqrt{c} \Theta(c)+\sum_{\text {bolts }} \frac{A}{4} .
\end{aligned}
$$

The final term in the volume integral can be decomposed into a surface and volume component. This yields

$$
\begin{aligned}
I^{\prime}= & -\frac{\beta}{16 \pi} \int_{\Xi} d^{3} x \sqrt{\gamma}\left[R(\gamma)-2(D \sigma)^{2}+\frac{1}{8} e^{-4 \sigma}(D \psi)^{2}\right] \\
& +\frac{\beta}{64 \pi} \int_{\partial \Xi} d^{2} x \sqrt{c} \psi e^{-4 \sigma} v^{i} D_{i} \psi-\frac{\beta}{8 \pi} \int_{\infty} d^{2} x \sqrt{c} \Theta(c)+\sum_{\text {bolts }} \frac{A}{4},
\end{aligned}
$$

which is a three-dimensional Einstein gravity theory coupled to the fields $\sigma$ and $\psi$. Note that $\psi$ is only defined up to a constant, but this constant will vanish when the background subtraction is made. If we define the field

$$
V=e^{2 \sigma}
$$

then we can rewrite the action as

$$
\begin{aligned}
I^{\prime}= & -\frac{\beta}{16 \pi} \int_{\Xi} d^{3} x \sqrt{\gamma}\left[R(\gamma)-\frac{1}{2 V^{2}}(D V)^{2}+\frac{1}{8 V^{2}}(D \psi)^{2}\right] \\
& +\frac{\beta}{64 \pi} \int_{\partial \Xi} d^{2} x \sqrt{c} \frac{1}{V^{2}} \psi v^{i} D_{i} \psi-\frac{\beta}{8 \pi} \int_{\infty} d^{2} x \sqrt{c} \Theta(c)+\sum_{\text {bolts }} \frac{A}{4} .
\end{aligned}
$$

Using the action (3.24) we can derive the equations of motion for $\gamma_{i j}, V$ and $\psi$,

$$
\begin{aligned}
R_{i j}-\frac{1}{2} \gamma_{i j} R & =\frac{1}{16 V^{2}}\left[8\left(D_{i} V\right)\left(D_{j} V\right)-4(D V)^{2}-2\left(D_{i} \psi\right)\left(D_{j} \psi\right)+(D \psi)^{2}\right] \\
V D^{2} V-3(D V)^{2} & =\frac{1}{4}(D \psi)^{2} \\
D^{i}\left[\frac{1}{V^{2}} D_{i} \psi\right] & =0 .
\end{aligned}
$$

The equation for $\gamma_{i j}$ implies that the volume term vanishes, while the equation for $\psi$ implies that there is a conserved quantity, the nut charge,

$$
\mathcal{N}=-\frac{1}{16 \pi} \int_{S^{2}} d^{2} x \sqrt{c} \frac{1}{V^{2}} u^{i} \partial_{i} \psi
$$

where $S^{2}$ is any topological two sphere, and $u^{i}$ is an outward pointing normal (i.e., at infinity $u^{i}=v^{i}$, while on the fixed point sets, $\left.u^{i}=-v^{i}\right)$. The nut charge is the same for all two-surfaces which are in the same cohomology class. We also know (by applying the field equation for $V$ ) that the nut potential must be constant on the fixed point set boundary. Thus, if we eliminate the volume term and decompose the integral on the boundary of the fixed point set into a nut potential and nut charge term, then we obtain

$$
I^{\prime}=\frac{\beta}{64 \pi} \int_{\infty} d^{2} x \sqrt{c} \frac{1}{V^{2}} \psi v^{i} D_{i} \psi-\frac{\beta}{8 \pi} \int_{\infty} d^{2} x \sqrt{c} \Theta(c)+\sum_{\text {bolts }} \frac{A}{4}+\frac{\beta}{4} \sum_{\mathrm{fps}} \psi \mathcal{N} .
$$


This is the most general form of the action, applicable to any boundary conditions. If we now restrict ourselves to ALL boundary conditions, then we can simplify the action further. As shown in appendix B, the surface integrals at infinity will exactly cancel in the background subtraction. Thus, the action becomes

$$
\bar{I}=\frac{\beta}{4} \sum_{\mathrm{fps}} \psi \mathcal{N}+\sum_{\text {bolts }} \frac{A}{4},
$$

so that there is a nonzero contribution from both the the area of the bolts, and the nut charge and potential of the nuts and bolts.

We now want to consider what happens to the action under various transformations. First, we want to consider a gauge transformation,

$$
\tau \rightarrow \tau+2 \lambda\left(x^{j}\right)
$$

which preserves the isometry, and hence the fixed point set. The orbit space metric and scalar field are also unaffected by this transformation, but the vector potential transforms as

$$
A_{i} \rightarrow A_{i}+\lambda_{, i}
$$

However, when we take the exterior derivative of $A_{i}$ to get the field strength $F_{i j}$, then the $\lambda$ dependent term, $\lambda_{,[i j]}$ is clearly zero. Thus, both the nut charge and potential are gauge invariant. Hence, each term in equation (3.29) for the action is separately gauge invariant.

We see that a dilation of the scalar fields,

$$
\psi \rightarrow b \psi \quad \text { and } \quad V \rightarrow b V
$$

leaves the action invariant, and hence gives rise to a conserved current, the dilation current,

$$
J_{i}=\frac{1}{V} D_{i} V-\frac{\psi}{4 V^{2}} D_{i} \psi
$$

Since $D_{i} J^{i}=0$, the flux of the dilation current through the boundary of $\Xi$ must vanish,

$$
\int_{\partial \Xi} d^{2} x \sqrt{c} v^{i} J_{i}=0
$$

Consider the flux of the dilation current through the fixed point sets

$$
\frac{\beta}{16 \pi} \int_{\mathrm{fps}} d^{2} x \sqrt{c} v^{i} J_{i}=\frac{\beta}{16 \pi} \int_{\mathrm{fps}} d^{2} x \sqrt{c}\left[v^{i} \frac{1}{V} D_{i} V-\frac{\psi}{4 V^{2}} v^{i} D_{i} \psi\right] .
$$

The first term is equal to negative one-quarter the area of the fixed point set, while the second term is negative one-quarter the nut charge times the nut potential (times $\beta$ ),

$$
\frac{\beta}{16 \pi} \int_{\mathrm{fps}} d^{2} x \sqrt{c} v^{i} J_{i}=-\frac{1}{4} \sum_{\mathrm{bolts}} A-\frac{\beta}{4} \sum_{\mathrm{fps}} \psi \mathcal{N} .
$$

However, in the case of ALL metrics, this is simply minus the action of the spacetime, so 


$$
\bar{I}=-\frac{\beta}{16 \pi} \int_{\mathrm{fps}} d^{2} x \sqrt{c} v^{i} J_{i},
$$

and hence we can convert the action into an integral at infinity,

$$
\bar{I}=\frac{\beta}{16 \pi} \int_{\infty} d^{2} x \sqrt{c} v^{i} J_{i}=\frac{\beta}{16 \pi} \int_{\infty} d^{2} x \sqrt{c} \frac{1}{V} v^{i} D_{i} V
$$

where we have used the fact that the integral involving the nut potential must vanish at infinity for ALE and ALF spaces. This expression for the action is related to the Komar mass of the spacetime,

$$
\bar{I}=\frac{1}{2} \beta M_{\text {Komar }},
$$

where

$$
M_{\mathrm{Komar}}=\frac{1}{8 \pi} \int_{\infty} d^{2} \Sigma_{\mu \nu} \nabla^{\mu} K^{\nu}=\frac{1}{8 \pi} \int_{\infty} d^{2} x \sqrt{c} \frac{1}{V} v^{i} D_{i} V
$$

This yields a generalized Smarr formula for the instanton,

$$
M_{\text {Komar }}=\frac{1}{4 \pi} \sum_{\text {bolts }} \kappa A+\frac{1}{2} \sum_{\mathrm{fps}} \psi \mathcal{N},
$$

where $\kappa=2 \pi / \beta$ is the surface gravity of the bolt.

\section{MISNER STRINGS AND THE NUT CHARGE}

A Misner string is a coordinate singularity which can be considered as a manifestation of a non-trivial topological twisting of the manifold. This twisting is parameterized by a topological term, the nut charge, which was defined in equation (3.28). That is, there will be Misner strings in the spacetime if and only if there is a nonzero source of nut charge (although the net nut charge of the spacetime may be zero).

The existence of a Misner string was first pointed out by Misner in his paper on the Lorentzian Taub-NUT spacetime [16]. The Lorentzian Taub-NUT solution contains a coordinate singularity running along one axis. Misner noticed that he could remove the coordinate singularity by using different time coordinates near the north and south poles. However, this coordinate transformation made the time periodic.

In the orbit space $\Sigma$, the Misner string is a line such that the integral of $A_{i}$ around a closed loop which encircles the string does not vanish as the area of loop is taken to zero - it is the Dirac string for the Kaluza-Klein vector potential $A_{i}$. The curvature is well-behaved along the string, implying that it is only a coordinate singularity. However, it is impossible to remove the singularity with coordinates which are adapted to the isometry - a gauge transformation which preserves the isometry, as given by equation (3.31), simply moves the singularity to another location (and doesn't affect the nut charge). But, by considering two coordinate charts (which have the string in different locations) it is possible to obtain a non-singular atlas for the manifold. 
In equation (3.28), the nut charge of a two-surface is expressed in terms of the nut potential, $\psi$. Instead, we could write the nut charge in terms of the field strength $F_{i j}$,

$$
\mathcal{N}=-\frac{1}{4 \pi} \int_{\mathcal{S}} \mathbf{F}
$$

where $\mathcal{S}$ is any topological two-sphere in the orbit space. Since $\mathbf{F}$ is related to the first Chern form, $\mathbf{c}_{\mathbf{1}}=-2 \beta^{-1} \mathbf{F}$, we can also write the nut charge in terms of the first Chern number, $C_{1}$ (which is an integer),

$$
\mathcal{N}=\frac{1}{8 \pi} \beta C_{1}
$$

Any surfaces $\mathcal{S}_{1}$ and $\mathcal{S}_{2}$ that can be continuously deformed into one another (i.e., belong to the same second homology class) will have the same nut charge. This is because the fixed points of $\xi$, which determine the internal boundaries of $\Xi$ (and hence the second homology classes), act as sources and sinks for the nut charge. We can think physically of nut charge current as flowing into or out of the boundaries around the fixed points, and along the Misner strings (which then must terminate on a boundary of $\Xi$ - either at a fixed point set or at infinity). The nut charge of a two-surface is therefore equal to the current carried out of the two-surface by the Misner strings. Thus, a nut-antinut pair, connected by a Misner string, will not provide any net nut charge (as measured by a two-surface at infinity), since the current will be entirely contained in the Misner string connecting the two. Only fixed points with Misner strings which extend to infinity will have a net nut charge.

The nut charge, as presented here, does not need to be quantized if we let the periodicity of the fibres vary, since it is only the value $C_{1}=8 \pi \mathcal{N} \beta^{-1}$ which is quantized. However, a quantization condition is obtained if the periodicity of the fibres is fixed. This will occur if the instanton is treated as the spacelike part of a five-dimensional theory (with a trivial timelike direction) and the $U(1)$ isometry is used to perform a Kaluza-Klein reduction to four dimensions. The periodicity of the fibre is related to the electric charge of the resulting Kaluza-Klein theory, and hence the nut charge, which can be interpreted as a corresponding magnetic charge, can be quantized in terms of the electric charge. For a discussion of this interpretation with respect to the Taub-NUT and the Kerr-Taub-Bolt instantons, see [17].

We now calculate the nut charge and nut potential for our example spacetimes and verify that equation (3.30) gives the correct value for the action.

\section{A. Examples}

The Kerr instanton, although it has no net nut charge, has a more complicated structure than either the Taub-Bolt or Eguchi-Hanson metrics, even though they do have a net nut chanrge. This is because Kerr has a nut-antinut pair. We can see this by considering the vector potential,

$$
\mathbf{A}=-\frac{a M r \sin ^{2} \theta}{r^{2}-2 M r-a^{2}+a^{2} \sin ^{2} \theta}
$$

and observing that in the orbit space, the fixed point sets at $r=r_{+}$and $\theta=0, \pi$ are joined by a Misner string, which is the horizon collapsed down to one dimension. On the Misner string, $r=r_{+}$, and hence 


$$
\mathbf{A}=-\frac{M r_{+}}{a} .
$$

Thus, the current flowing along the Misner string is

$$
-\frac{1}{4 \pi} \int_{0}^{2 \pi / p} d \phi A_{\phi}=\frac{M r_{+}}{2 a p}=\frac{1}{4 \Omega p},
$$

where we recall that the $\phi$ only runs from 0 to $2 \pi / p$. But, this gives the nut charges of the the nut-antinut pair,

$$
\mathcal{N}_{\text {np }}=\frac{1}{4 \Omega p} \quad \text { and } \quad \mathcal{N}_{\mathrm{sp}}=-\frac{1}{4 \Omega p},
$$

where $n p$ and $s p$ stand for the north and south poles respectively. The nut potential is

$$
\psi=\frac{4 a M \cos \theta}{r^{2}-a^{2} \cos ^{2} \theta} .
$$

Thus, if we evaluate it on the nut and antinut, we obtain

$$
\psi_{n p}=4 M \Omega \quad \text { and } \quad \psi_{s p}=-4 M \Omega,
$$

so that the nut and antinut make identical contributions to the action. Hence, remembering that the periodicity of $\tau$ is $2 \pi \gamma p$, we see that the action is

$$
I=2 \times \frac{1}{4}(2 \pi \gamma p)(4 M \Omega) \frac{1}{4 \Omega p}=\pi \gamma M,
$$

as calculated previously. Note that since the background has no fixed point set, there is no background subtraction.

The Taub-NUT and Taub-Bolt metrics are both of the same form (where $V$ is different for the two metrics), and have field strength

$$
\mathbf{F}=-N \sin \theta \mathbf{d} \theta \wedge \mathbf{d} \phi .
$$

If we integrate the field strength over any two-sphere surrounding the internal boundary (due to the fixed point set), we obtain $\mathcal{N}=N$. Note that in the Taub-NUT case, the nut charge is due to the presence of a nut, while in the Taub-Bolt case, it arises from a bolt.

We can calculate $\psi$ for the Taub-Bolt and Taub-NUT cases,

$$
\psi(r)=\frac{(4 r-5 N) N}{(r-N)(r+N)} \quad \text { and } \quad \tilde{\psi}(r)=\frac{4 N}{r+N},
$$

where we have normalized the potentials such that they vanish at infinity. If we evaluate them on the fixed point sets of the two metrics we obtain

$$
\psi(2 N)=1, \quad \text { and } \quad \tilde{\psi}(N)=2 .
$$

Thus, if we substitute the nut charge and nut potential into equation (3.30), we obtain an action of 


$$
\bar{I}=\frac{1}{4} 8 \pi N^{2}-\frac{1}{4} 16 \pi N^{2}+\frac{1}{4} 12 \pi N^{2}=\pi N^{2} .
$$

For the Eguchi-Hanson metric (2.35), we see that the field strength is

$$
\mathbf{F}=-2 N \sin \theta \mathbf{d} \theta \wedge \mathbf{d} \phi .
$$

If we integrate this over any two-sphere surrounding the internal boundary, we get $\mathcal{N}=2 N$. This nut charge is due to the bolt at $r=N$. The nut potentials are

$$
\psi=-\frac{1}{32} \frac{r^{4}+N^{4}}{r^{2} N^{2}}, \quad \text { and } \quad \tilde{\psi}=-\frac{1}{32} \frac{r^{4}+M^{4}}{r^{2} N^{2}}
$$

Note that although these terms diverge at infinity, the difference between them goes to zero, as explicitly shown in appendix B for any ALE metric. If we evaluate the potentials on the bolt, then we obtain

$$
\psi(N)=-\frac{1}{16} \quad \text { and } \quad \tilde{\psi}(M)=-\frac{M^{2}}{16 N^{2}}
$$

Thus, the action is

$$
I=\frac{1}{4}\left[\pi N^{2}-\pi M^{2}+16 \pi N^{2} \frac{M^{2}-N^{2}}{16 N^{2}}\right]=0 .
$$

\section{CONCLUSIONS}

We have examined some of the properties of four-dimensional instantons which have a nut charge, and found that they contain additional complications that the simpler black hole metrics which have been studied up to now do not possess. If we dimensionally reduce the action of the instanton along the isometry, we see that it cannot be described purely in terms of the area of the fixed point set, but must include a contribution from the nut charge and nut potential of the instanton, as well as from a surface integral at infinity. The action is given by equation (3.29)

$$
I^{\prime}=\frac{\beta}{64 \pi} \int_{\infty} d^{2} x \sqrt{c} \frac{1}{V^{2}} \psi v^{i} D_{i} \psi-\frac{\beta}{8 \pi} \int_{\infty} d^{2} x \sqrt{c} \Theta(c)+\sum_{\text {bolts }} \frac{A}{4}+\frac{\beta}{4} \sum_{\mathrm{fps}} \psi \mathcal{N} .
$$

Each term in this expression was found to be invariant under the gauge transformation $\tau \rightarrow \tau+2 \lambda\left(x^{j}\right)$. In the case of ALL metrics, it was also shown that the surface integrals vanish when a background subtraction is made, so that the action reduces to a simple sum over fixed point set quantities that includes both area and nut terms (equation (3.30),

$$
\bar{I}=\frac{\beta}{4} \sum_{\mathrm{fps}} \psi \mathcal{N}+\sum_{\text {bolts }} \frac{A}{4} .
$$

By considering the behaviour of the action under a dilation of the scalar fields $V$ and $\psi$, we can rewrite the action for ALL spaces in terms of the Komar mass, 


$$
\bar{I}=\frac{1}{2} \beta M_{\text {Komar }}
$$

which then yields a generalized Smarr formula,

$$
M_{\mathrm{Komar}}=\frac{1}{4 \pi} \sum_{\text {bolts }} \kappa A+\frac{1}{2} \sum_{\mathrm{fps}} \psi \mathcal{N}
$$

As mentioned in the introduction, the effect of nut charge on the entropy of instantons is the underlying motivation for this paper. While we have not directly addressed this issue, we have proved some results about the action of instantons with nut charge that will prove useful in tackling the question of entropy [3].

\section{ACKNOWLEDGEMENTS}

I would like to thank S.W. Hawking for suggesting the problem and for useful discussion. The Association of Commonwealth Universities and the Natural Sciences and Engineering Research Council of Canada have provided financial support.

\section{APPENDIX A: EXTRINSIC CURVATURE ON A BOLT}

We are interested in evaluating the trace of the extrinsic curvature of a bolt embedded in the three dimensional orbit space $\Xi$. Near the bolt, we can assume that the metric is given by

$$
d s^{2}=V\left(x^{i}\right)\left(d \tau+2 A_{i} d x^{i}\right)^{2}+\frac{C^{2}\left(x^{i}\right)}{V\left(x^{i}\right)} d r^{2}+p_{a b} d x^{a} d x^{b}
$$

where the periodicity of $\tau$ is $\beta, p_{a b} d x^{a} d x^{b}$ is the (nonvanishing) metric on a topological two-sphere (which becomes the bolt at the zero of $V$, which is assumed to be a constant $r$ surface), and $C\left(x^{i}\right)$ is constant on the bolt. We are interested in deriving the condition on $\beta$ which is necessary to avoid a conical singularity in the $\tau-r$ plane. To this end, we can restrict our attention to the two dimensional metric

$$
d s^{2}=V\left(x^{i}\right) d \tau^{2}+\frac{C^{2}\left(x^{i}\right)}{V\left(x^{i}\right)} d r^{2} .
$$

We now want to express this as a polar coordinate system. If we introduce a new angular coordinate, $\psi=2 \pi \tau / \beta$, which has period $2 \pi$, then the metric is

$$
d s^{2}=\frac{\beta^{2}}{(2 \pi)^{2}} V\left(x^{i}\right) d \psi^{2}+\frac{C^{2}\left(x^{i}\right)}{V\left(x^{i}\right)} d r^{2} .
$$

We can move the origin of the coordinate system to $R=0$ by introducing the coordinate

$$
R=\frac{\beta}{2 \pi} \sqrt{V}
$$


The metric is then

$$
d s^{2}=R^{2} d \psi^{2}+\frac{(4 \pi)^{2}}{\beta^{2}} \frac{C^{2}}{\left(V^{\prime}\right)^{2}} d R^{2}
$$

where we have ignored terms which are not in the $\psi-r$ plane, and $V^{\prime}$ indicates a derivative with respect to $r$. In order to avoid a conical singularity as $R \rightarrow 0$ (that is, as we approach the bolt), we need

$$
\frac{V^{\prime}}{C}=\frac{4 \pi}{\beta}
$$

Now we want to use this condition on $V^{\prime}$ to evaluate the trace of the extrinsic curvature on the bolt. The orbit space variables are

$$
\begin{aligned}
\gamma_{i j} d x^{i} d x^{j} & =C^{2} d r^{2}+V p_{i j} d x^{i} d x^{j} \\
\sqrt{\gamma} & =C V \sqrt{p} \\
v^{r} & =-C^{-1}
\end{aligned}
$$

where $v^{i}$ is the unit inward-pointing normal to the bolt. If we calculate the trace of the extrinsic curvature of the bolt, we obtain

$$
\begin{aligned}
\sqrt{c} \Theta(c) & =\frac{\sqrt{c}}{\sqrt{\gamma}} \frac{d}{d r}\left(v^{r} \sqrt{\gamma}\right) \\
& =-\frac{1}{C} \frac{\partial}{\partial r}[V \sqrt{p}] \\
& =-\left[\frac{V^{\prime}}{C} \sqrt{p}+\frac{V p^{\prime}}{2 C \sqrt{p}}\right] .
\end{aligned}
$$

But, we are evaluating this term on the bolt, where we have conditions on both $V$ and $V^{\prime}$. That is, $V$ vanishes on the bolt, and $V^{\prime}$ is given by equation (A6). Thus, the trace of the extrinsic curvature is simply

$$
\sqrt{c} \Theta(c)=-\frac{4 \pi}{\beta} \sqrt{p}
$$

Hence, if we integrate the trace of the extrinsic curvature over the bolt, we see that

$$
-\frac{\beta}{8 \pi} \int_{\text {bolt }} d^{2} x \sqrt{c} \Theta(c)=\frac{\beta}{8 \pi} \int_{\text {bolt }} d^{2} x \frac{4 \pi}{\beta} \sqrt{p}=\frac{1}{2} \int_{\text {bolt }} d^{2} x \sqrt{p}
$$

where the final integral is simply the area of the bolt. Thus,

$$
-\frac{\beta}{8 \pi} \int_{\text {bolt }} d^{2} x \sqrt{c} \Theta(c)=\frac{A}{2}
$$

where $A$ is the area of the bolt. 


\section{APPENDIX B: SURFACE INTEGRALS AT INFINITY}

In this appendix we are concerned with integrals in the orbit space, over the boundary at infinity, that arise in the dimensionally reduced equation for the action (3.29). There are two integrals: a curvature integral,

$$
I_{c}=\int_{\infty} d^{2} x \sqrt{c}[\Theta(c)-\Theta(\tilde{c})]
$$

and a nut integral,

$$
I_{\psi}=\int_{\infty} d^{2} x \sqrt{c} \frac{1}{V^{2}}\left(\psi v^{i} D_{i} \psi-\tilde{\psi} \tilde{v}^{i} \tilde{D}_{i} \tilde{\psi}\right)
$$

We will consider these integrals for metrics which have AF, ALF and ALE (i.e., ALL) asymptotics, as defined in section 11 .

Beginning with the AF class, we write the asymptotic metric (2.8) in Kaluza-Klein form,

$$
d s^{2} \sim\left(1-\frac{2 M}{r}\right) d \tau^{2}+\left(1-\frac{2 M}{r}\right)^{-1} d \mathcal{E}_{3}^{2}+\mathcal{O}\left(r^{-2}\right)
$$

and hence, up to $\mathcal{O}\left(r^{-2}\right)$, the orbit space metric is simply three dimensional Euclidean space. Calculating the trace of the extrinsic curvature of a surface of constant radius $R$, we find that

$$
\sqrt{c} \Theta(c) \sim 2 R \sin \theta+\mathcal{O}\left(R^{-1}\right)
$$

In order to calculate the curvature integral (B1), we also need the trace of the extrinsic curvature of the boundary at infinity in the background metric. From (2.11), the matched background metric is

$$
d \tilde{s}^{2} \sim\left(1-\frac{2 M}{R}\right) d \tau^{2}+\left(1-\frac{2 M}{R}\right)^{-1} d \mathcal{E}_{3}^{2}+\mathcal{O}\left(R^{-2}\right)
$$

and hence the orbit space metric is also three dimensional Euclidean space. Thus, the calculation of the trace fo the extrinsic curvature of the constant radius $R$ surface will be identical to the general AF calculation done above. So,

$$
\sqrt{c} \Theta(\tilde{c}) \sim 2 R \sin \theta+\mathcal{O}\left(R^{-1}\right)
$$

and therefore

$$
I_{c}^{\mathrm{AF}}=\int_{\infty} d^{2} x \sqrt{c}[\Theta(c)-\Theta(\tilde{c})]=0
$$

We now want to calculate the nut integral (B2) for an AF metric. Since the asymptotic form of the vector potential is $\mathcal{O}\left(r^{-2}\right)$, the nut potential equation (3.20) tells us that on a surface of constant radius $R$,

$$
\psi \sim \mathcal{O}\left(R^{-2}\right) \quad \text { and } \quad \partial_{r} \psi \sim \mathcal{O}\left(R^{-3}\right)
$$


where we have set the arbitrary constant equal to zero. The asymptotic behaviour of the nut integrand is therefore

$$
\sqrt{c} \frac{1}{V^{2}} \psi v^{i} D_{i} \psi \sim \mathcal{O}\left(R^{-3}\right)
$$

The nut potential of the background is clearly constant (since the vector potential vanishes identically), and hence can be set to zero. Thus, the nut integral will vanish as we take $R$ to infinity,

$$
I_{\psi}^{\mathrm{AF}}=\int_{\infty} d^{2} x \sqrt{c} \frac{1}{V^{2}}\left(\psi v^{i} D_{i} \psi-\tilde{v}^{i} \tilde{D}_{i} \tilde{\psi}\right)=0
$$

If we now turn our attention to the ALF class and write the asymptotic metric (2.9) in Kaluza-Klein form,

$$
d s^{2} \sim\left(1-\frac{2 M}{r}\right)[d \tau+2 a N \cos \theta d \phi]^{2}+\left(1-\frac{2 M}{r}\right)^{-1} d \mathcal{E}_{3}^{2}+\mathcal{O}\left(r^{-2}\right)
$$

then we see (again, up to $\mathcal{O}\left(r^{-2}\right)$ ) that the orbit space metric is three-dimensional Euclidean space. Since this is the same as in the AF case, the trace of the extrinsic curvature of a constant radius $R$ surface must be the same,

$$
\sqrt{c} \Theta(c) \sim 2 R \sin \theta+\mathcal{O}\left(R^{-1}\right)
$$

The matched background metric (2.14) has Kaluza-Klein form

$$
\begin{aligned}
d \tilde{s}^{2} \sim & \left(1-\frac{2 N}{r}-\frac{2(M-N)}{R}\right)[d \tau+2 a N \cos \theta d \phi]^{2} \\
& +\left(1-\frac{2 N}{r}-\frac{2(M-N)}{R}\right)^{-1} d \mathcal{E}_{3}^{2}+\mathcal{O}\left(r^{-2}\right)
\end{aligned}
$$

and hence its orbit space metric is also three-dimensional Euclidean space. Thus, the trace of the extrinsic curvature of the boundary at large radius $R$ is the same as the trace of the extrinsic curvature in the previous three cases. The curvature integral will therefore vanish for ALF metrics,

$$
I_{c}^{\mathrm{ALF}}=\int_{\infty} d^{2} x \sqrt{c}[\Theta(c)-\Theta(\tilde{c})]=0 .
$$

We now want to calculate the nut integral. Using the one-form potential, $A_{\phi}=a N \cos \theta$, we can solve the the nut potential equation and find that on our boundary

$$
\psi \sim \frac{4 a N}{R}+\mathcal{O}\left(R^{-2}\right) \quad \text { and } \quad \partial_{r} \psi \sim-\frac{4 a N}{R^{2}}+\mathcal{O}\left(R^{-3}\right)
$$

Hence the asymptotic behaviour of the nut integrand is

$$
\sqrt{c} \frac{1}{V^{2}} \psi v^{i} D_{i} \psi \sim \mathcal{O}\left(R^{-1}\right) .
$$


Clearly, the asymptotic behaviour of the background nut potential, and hence the background nut integrand, will be the same, i.e. $\mathcal{O}\left(R^{-1}\right)$, and thus the nut integral (B2) will be $\mathcal{O}\left(R^{-1}\right)$ at large radius $R$, and so will vanish at infinity,

$$
I_{\psi}^{\mathrm{ALF}}=\int_{\infty} d^{2} x \sqrt{c} \frac{1}{V^{2}}\left(\psi v^{i} D_{i} \psi-\tilde{v}^{i} \tilde{D}_{i} \tilde{\psi}\right)=0
$$

Finally, we want to tackle the ALE metrics. We write the asymptotic form of the metric (2.10) in Kaluza-Klein form

$$
\begin{aligned}
d s^{2} \sim & \left(1+\frac{M}{r^{4}}\right) \frac{r^{2}}{(4 a N)^{2}}[d \tau+2 a N \cos \theta d \phi]^{2}+\frac{(4 a N)^{2}}{r^{2}\left(1+M / r^{4}\right)} \\
& \times\left\{\left(1+\frac{2 M}{r^{4}}\right) \frac{r^{2}}{(4 a N)^{2}}\left[d r^{2}+\frac{r^{2}}{4}\left(d \theta^{2}+\sin ^{2} \theta d \phi^{2}\right]\right\}+\mathcal{O}\left(r^{-5}\right) .\right.
\end{aligned}
$$

The orbit space metric is

$$
d s_{3}^{2} \sim\left(1+\frac{2 M}{r^{4}}\right)\left[d r^{2}+\frac{r^{2}}{4}\left(d \theta^{2}+\sin ^{2} \theta d \phi^{2}\right)\right]+\mathcal{O}\left(r^{-5}\right) .
$$

We can then calculate the trace of the extrinsic curvature of the constant radius $R$ surface,

$$
\sqrt{c} \Theta(c) \sim \frac{R^{2}}{4 a N} \sin \theta+\mathcal{O}\left(R^{-2}\right)
$$

To obtain the trace of the extrinsic curvature of this surface in the background metric (2.15), we note that the background metric is simply the $M=0$ case of the general ALE metric. Thus, up to $\mathcal{O}\left(R^{-2}\right)$, the trace of extrinsic curvatures will be the same, and hence the curvature integral will vanish,

$$
I_{c}^{\mathrm{ALE}}=\int_{\infty} d^{2} x \sqrt{c}[\Theta(c)-\Theta(\tilde{c})]=0 .
$$

Last, but not least, we need to calculate the nut potential for an ALE metric. Substituting the vector potential, $A_{\phi}=a N \cos \theta$, into the nut potential equation yields

$$
\psi \sim-\frac{2}{(4 a N)^{2}}\left(r^{2}-\frac{M}{r^{2}}\right)+\mathcal{O}\left(r^{-3}\right) .
$$

From this, we can evaluate the integrand of the nut integral over the boundary,

$$
\sqrt{c} \frac{1}{V^{2}} \psi v^{i} D_{i} \psi \sim \frac{2}{4 a N} R^{2} \sin \theta+\mathcal{O}\left(R^{-2}\right) .
$$

Just as before, we can obtain the background value by simply setting $M=0$. This yields a background nut integrand which is identical (up to $\mathcal{O}\left(R^{-2}\right)$ ) with equation (B23), and hence the nut integral will vanish as we take the constant radius surface to infinity,

$$
I_{\psi}^{\mathrm{ALE}}=\int_{\infty} d^{2} x \sqrt{c} \frac{1}{V^{2}}\left(\psi v^{i} D_{i} \psi-\tilde{v}^{i} \tilde{D}_{i} \tilde{\psi}\right)=0 .
$$

In summary, we have shown that that the curvature and nut integrals given by equations (B1) and (B2) vanish for ALL metrics. This justifies the simplification of the action formula from equation (3.29) to (3.30) for this class of metrics. 


\section{REFERENCES}

[1] A. Strominger and C. Vafa, "Microscopic Origin of the Bekenstein-Hawking Entropy", hep-th/9601029.

[2] G.W. Gibbons and S. W. Hawking, "Action Integrals and Partition Functions in Quantum Gravity", Phys. Rev. D15, 2752 (1977).

[3] S. W. Hawking and C. J. Hunter, "The Entropy of Instantons with Nut Charge", paper in preparation.

[4] S. W. Hawking, "Gravitational Instantons", Phys. Lett. 60A, 81 (1977).

[5] G. W. Gibbons and S. W. Hawking "Gravitational Multi-Instantons", Phys. Lett. 78B, 430 (1978).

[6] G. W. Gibbons and S. W. Hawking, "Classification of Gravitational Instanton Symmetries", Comm. Math. Phys. 66, 291 (1979).

[7] S. W. Hawking and G. T. Horowitz, "The Gravitational Hamiltonian, Action, Entropy and Surface Terms", Class. Quant. Grav. 13, 1487 (1996).

[8] S. W. Hawking and C. J. Hunter, "The Gravitational Hamiltonian in the Presence of Non-Orthogonal Boundaries", Class. Quant. Grav. 13, 2735 (1996).

[9] G. W. Gibbons, C. N. Pope, "The Positive Action Conjecture and Asymptotically Euclidean Metrics in Quantum Gravity", Comm. Math. Phys. 66, 267 (1979).

[10] R.M. Schoen and S. Yau "Proof of the Positive Action Conjecture in Quantum Relativity", Phys. Rev. Lett. 42, 547 (1979).

[11] D. N. Page, "Taub-NUT Instanton with an Horizon", Phys. Lett. 78B, 249 (1978).

[12] S. W. Hawking and C. N. Pope, "Generalized Spin Structures in Quantum Gravity", Phys. Lett. 73B, 42 (1978).

[13] J. P. Gauntlett, G. W. Gibbons, G. Papadopoulous and P. K. Townsend, "Hyper-Kähler Manifolds and Multiply Intersecting Branes", hep-th/9702202

[14] G. W. Gibbons and M. J. Perry, "New gravitational instantons and their interactions", Phys. Rev. D22, 313 (1980).

[15] T. Eguchi and A. J. Hanson, "Asymptotically Flat Self-Dual Solutions to Euclidean Gravity", Phys. Lett. 74B, 249 (1978).

[16] C. W. Misner, "The Flatter Regions of Newman, Unti, and Tamburino's Generalized Schwarzschild Space", Journal of Math. Phys. 4, 924 (1963).

[17] D. J. Gross and M. J. Perry, "Magnetic Monopoles in Kaluza-Klein Theories", Nucl. Phys. B226, 29 (1983). 